\title{
Faraday-rotation fluctuation spectroscopy with static and oscillating magnetic fields
}

\author{
Matthias Braun and Jürgen König \\ Institut für Theoretische Physik III, Ruhr-Universität Bochum, 44780 Bochum, Germany
}

(Dated: September 3, 2018)

\begin{abstract}
By Faraday-rotation fluctuation spectroscopy one measures the spin noise via Faraday-induced fluctuations of the polarization plane of a laser transmitting the sample. In the fist part of this paper, we present a theoretical model of recent experiments on alkali gas vapors and semiconductors, done in the presence of a static magnetic field. In a static field, the spin noise shows a resonance line, revealing the Larmor frequency and the spin coherence time $T_{2}$ of the electrons. Second, we discuss the possibility to use an oscillating magnetic field in the Faraday setup. With an oscillating field applied, one can observe multi-photon absorption processes in the spin noise. Furthermore an oscillating field could also help to avoid line broadening due to structural or chemical inhomogeneities in the sample, and thereby increase the precision of the spin-coherence time measurement.
\end{abstract}

PACS numbers: $72.70 .+\mathrm{m}$ 78.47.+p 76.60.Lz 78.67.-n

\section{INTRODUCTION}

The anticipation of an application in quantum information processing ${ }^{1.2}$ motivates the intensive research on spin dynamics and spin coherence, especially in semiconductors 3.4 .5 Beside orthodox magnetic resonance experiments,, 6.7 .8 mainly two optical measurement schemes for the spin dynamics are in use: studies of the Hanle effect, $, 9,10$ and time-resolved Faraday (or Kerr) rotation 11.12 The former relies on the decrease of photoluminescence polarization due to an external magnetic field, the latter on the dependence of the phase velocity of polarized light on the spin orientation in the sample. While the Hanle setup measures the spins of excited electrons, the Faraday setup does not require this excitation. That optical measurements are possible at all is a consequence of spin-orbit coupling.

The time-resolved Faraday rotation has proven to be a very precise experimental tool, capable to address the spin dynamics down to the ps time scale. However, the necessary time resolution for such experiments requires high experimental efforts, i.e. a Streak-camera system. Already in 1981, Aleksandrov and Zapassky experimentally demonstrated,, 13 that instead of measuring the Faraday-rotation in an alkali gas, one can also measure Faraday-rotation fluctuations to observe the spin dynamics. Thereby latter experimental setup which does not require a time-resolution at all. They applied a static external magnetic field and transmit linear polarized laser light through the sample, perpendicular to the field, see Fig 1 Due to the Faraday effect, the spin noise in the sample is mapped on the fluctuations of the laser polarization plane, and latter can easily be measured. The precession of the spins in the sample gives rise to a Lorentzian line in the power spectrum of the Faradayrotation fluctuations. The noise frequency of this line is therefore a direct measure of the Larmor frequency $\omega_{0}$, and the line width indicates the electron-spin coherence time $T_{2}$.

Recently Crooker et al ${ }^{14}$ presented an increase of the precision of this optical magnetic resonance experiment ${ }^{15}$

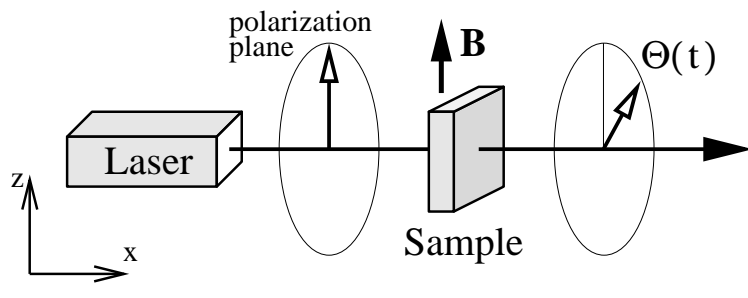

FIG. 1: A linear polarized laser is send through the sample. Due to spin-orbit coupling and the Faraday effect, the laser polarization plane rotates depending on the electron spins inside the sample. While the time averaged Faraday-rotation angle is zero, since there is no net magnetization collinear to the laser propagation direction, the Faraday-rotation fluctuations are finite.

capable to resolve even the different isotope lines in a $\mathrm{Ru}-$ bidium gas. On the other side Oestreich et al ${ }^{16}$ demonstrated that Faraday-rotation fluctuation spectroscopy can also be used in a solid-state environment: they addressed the electron spin precession in bulk GaAs.

In the following Sec. III we present an elementary theoretical description of these existing Faraday-rotation fluctuation experiments 13.14 .15 .16 (that involves a static magnetic field) within a density-matrix formulation. Then, we use this language to propose a different measurement scheme, namely to apply an oscillating magnetic field. In this case, the resonance will not appear at the (eventually locally varying) Larmor frequency, but at multiples of the oscillation frequency of the magnetic field. Therefore some sources of inhomogeneous broadening could be avoided.

\section{THEORETICAL DESCRIPTION}

Since Faraday rotation measures the spin component collinear with the laser propagation direction, depending on the optical selection rules of the sample, the fluctuation of the Faraday rotation is a direct measure of the 
transverse spin-spin correlation function

$$
S(t)=\left\langle\hat{s}_{\mathrm{X}}(t) \hat{s}_{\mathrm{X}}(0)+\hat{s}_{\mathrm{X}}(0) \hat{s}_{\mathrm{X}}(t)\right\rangle .
$$

The average of operators can be expressed by the trace $\left\langle\hat{s}_{\mathrm{X}}(t) \hat{s}_{\mathrm{X}}(0)\right\rangle=\operatorname{Tr}\left[\hat{s}_{\mathrm{X}}(t) \hat{s}_{\mathrm{X}}(0) \rho\right]$, where $\rho$ is the $\mathrm{SU}(2)$ density matrix describing one localized spin in the sample, and $\hat{s}$ is the $2 \times 2$ Pauli spin operator. The time evolution of the operator $\sigma_{x}$ in the Heisenberg picture reads $\hat{s}_{\mathrm{x}}(t)=(\hbar / 2) \exp \left[i / \hbar \int_{0}^{t} \hat{H}(\tau) d \tau\right] \sigma_{x} \exp \left[-i / \hbar \int_{0}^{t} \hat{H}(\tau) d \tau\right]$, if Hamiltonians at different times commute, which will be the case for a magnetic field with static or oscillating field strength. It is convenient to represent the correlator $S(t)$ as a diagram with the operators $\hat{s}_{\mathrm{X}}(0)$ and $\hat{s}_{\mathrm{X}}(t)$ placed on a Keldysh time contour $t_{\mathrm{K}}$, see Fig. 2 The

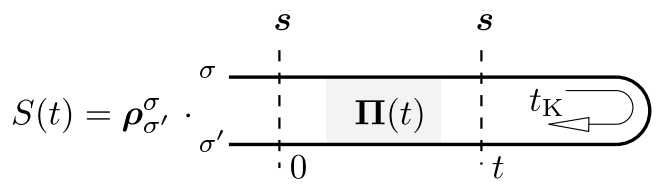

FIG. 2: Diagrammatic representation of $S(t)$. The operators $\hat{s}_{\mathrm{x}}$ are placed on the upper or lower branch of the time contour at time 0 and $t$, respectively.

spin states $\uparrow, \downarrow$ propagate along the upper time contour from the time 0 to $t$, and on the lower line from $t$ to 0 . This takes into account the Heisenberg time evolution of $\hat{s}_{\mathrm{X}}(t)$.

Instead of reading the time contour along $t_{\mathrm{K}}$ for the individual spin states of the Hilbert space, it is more intuitive to read the diagram from left to right, and interpret the double line of upper and lower Keldysh propagator as the time evolution of the whole density matrix $\rho_{\sigma^{\prime}}^{\sigma}$. We start with an initial density matrix at time 0 , and measure the spin state by the operator $s$. Then, the density matrix propagates from time 0 to time $t$, and the spin is measured again. From this perspective, Eq. (1) can be rewritten as

$$
S(t)=\frac{1}{2} \operatorname{Tr}[s \boldsymbol{\Pi}(t) s \rho]
$$

The (Liouville) operator $s$ accounts for placing $\hat{s}_{\mathrm{x}}$ at the upper or lower Keldysh contour, flipping the spin from $\sigma$ to $\bar{\sigma}$. By summing over both possibilities, the time symmetrization of Eq. (1) is taken into account 17 . The (Liouville) operator $s$ is a fourth-order tensor with $s_{\sigma^{\prime} \sigma^{\prime}}^{\sigma \bar{\sigma}}=s_{\sigma \bar{\sigma}}^{\sigma^{\prime} \sigma^{\prime}}=\hbar / 2$ and zero otherwise.

Between the two spin measurements at time 0 and time $t$, the propagation of the density matrix is described by $\Pi_{\sigma^{\prime} \sigma^{\prime}}^{\sigma \sigma}(t)=\exp \left\{-i / \hbar \int_{0}^{t}\left[\langle\sigma|\hat{H}(\tau)| \sigma\rangle-\left\langle\sigma^{\prime}|\hat{H}(\tau)| \sigma^{\prime}\right\rangle\right] d \tau\right\}$. Since we assume the magnetic field to be along the spin quantization axis, the states $\sigma, \sigma^{\prime}$ are eigenstates of the Hamilton operator, and no elements of $\Pi$ with different spin indices on the upper (or lower) propagator appear.

The formulation Eq. (2) offers the possibility to phenomenologically include transverse spin relaxation in the calculation. If the initial density matrix was in a diagonal state, the first spin operator $\hat{s}_{\mathrm{x}}$ brings it in a nondiagonal state $\rho_{\bar{\sigma}}^{\sigma}$. During the time evolution from 0 to $t$ non-diagonal density matrix elements decay exponentially with the time scale set by the spin-coherence time $T_{2}$. This is accounted for by multiplying $\Pi_{\bar{\sigma} \bar{\sigma}}^{\sigma \sigma}(t)$ with $\exp \left(-t / T_{2}\right)$. Such a exponential decay can for example be justified within a white noise model 18

\section{STATIC MAGNETIC FIELD}

To apply the formulation introduced above to the experiments presented in Ref. 13141516 , we set $H(t)=$ $-\omega_{0} \hat{s}_{\mathrm{z}}$ with $\omega_{0}=g \mu_{\mathrm{B}} B / \hbar$. Consequently, the propagator for a spin is then given by

$$
\Pi_{\sigma \sigma}^{\sigma \sigma}=1, \quad \Pi_{\downarrow \downarrow}^{\uparrow \uparrow}=e^{i \omega_{0} t-t / T_{2}}, \quad \Pi_{\uparrow \uparrow}^{\downarrow \downarrow}=e^{-i \omega_{0} t-t / T_{2}},
$$

and zero otherwise. The resulting power spectrum $S(\omega)=\int_{0}^{\infty} d t[\exp (-i \omega t)+\exp (i \omega t)] S(t)$ of the timedependent correlator $S(t)$ equals then

$$
S(\omega)=\frac{\hbar^{2}}{2}\left(\frac{T_{2}}{1+T_{2}^{2}\left(\omega-\omega_{0}\right)^{2}}+\frac{T_{2}}{1+T_{2}^{2}\left(\omega+\omega_{0}\right)^{2}}\right) .
$$

It shows a Lorentzian resonance centered at the Larmor frequency $\omega_{0}$, and the resonance width is given by $T_{2}$, comparable to a continuous-wave ESR experiment. We performed a fit to the experimental data of Oestreich et $a l^{16}$ in Fig. 3 .

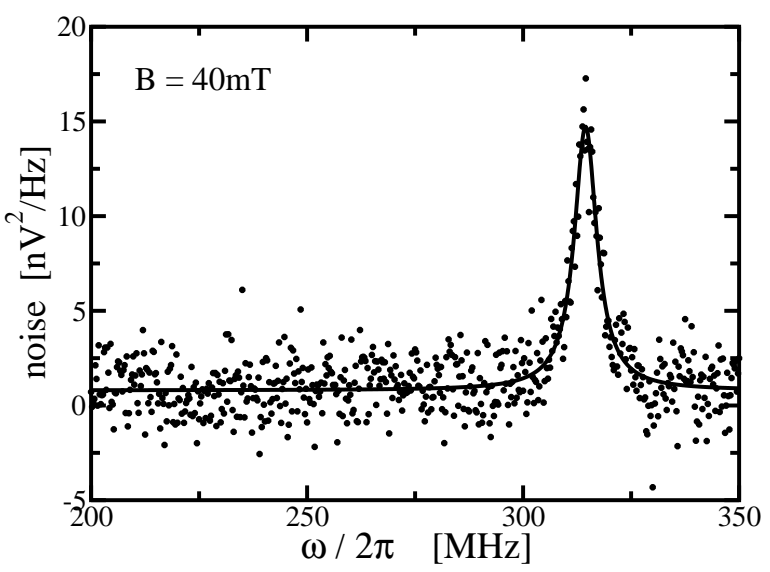

FIG. 3: Measured Faraday-rotation noise of a spin ensemble by Oestreich et al. ${ }^{16}$ together with a fit of a Lorentzian function as expected for a single spin, see Eq. (4).

The origin of the resonance can be understood by tracking the time evolution of the spin between two measurements. Here for simplicity, we consider only the spin component along the laser propagation direction, i.e., along the $x$-axis, since only this component determines the Faraday rotation of the laser polarization plane. Let 
us assume that at time $t=0$ the spin is aligned parallel to the direction of measurement, as shown in Fig. 4 The outcome of the first measurement therefore equals $s_{\mathrm{x}}=+\hbar / 2$. This spin then precesses in the static external magnetic field. If the time between first and second measurement is an integer times the time for a full revolution of the spin, the outcome of the second measurement will for certain be $s_{\mathrm{x}}=+\hbar / 2$, i.e., the results of the two measurements coincide. The probability to measure the same spin state again is reduced by spin relaxation / decoherence.

For half-integer multiples, the measurement results will have opposite signs. The spin-spin correlation function is, therefore, an oscillating function in time, which is exponentially damped due to decoherence. The corresponding power spectrum is a Lorentzian centered at the Larmor frequency $\omega_{0}$ with a width of $T_{2}$. This argument also holds, if the initial spin state is a coherent superposition of spin up and down, i.e., the initial spin is unimportant. In contrast to an ESR experiment, no net magnetization of the semiconductor sample is needed, which is reflected in the fact, that Eq. (44) is independent of the initial density matrix.
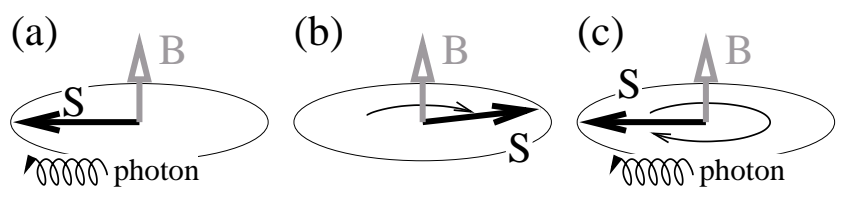

FIG. 4: Sketch of the electron spin dynamics in the Faraday setup with a static magnetic field. (a) At time $t=0$, the spin is measured. (b) Between the two measurement, the spin precesses in a static magnetic field $\boldsymbol{B}$. (c) If the second measurement takes place after a full revolution, it reproduces the outcome of the first measurement.

\section{OSCILLATING MAGNETIC FIELD}

We now turn to the case of an oscillating magnetic field along the $z$-direction. The difference to the case of a static magnetic field is that the spin precession between the two measurements can change its direction as a function of time. This is quite similar to the case in spin-echo resonance experiments ${ }^{6.7}$ After the first measurement the spin precesses in one direction but then stops and precesses back. After a full oscillation period of the external field, the spin will be just back at its starting point, see Fig. [5 If the second measurement takes place at this time, the outcome will most probable be equal to the first measurement, i.e., it will be correlated. The probability of equal spin measurements is decreased by decoherence.

Technically, we can describe the oscillating magnetic field by the time-dependent Hamiltonian

$$
H(t)=-\omega_{0} \cos (\gamma t+\phi) \cdot \hat{s}_{z}
$$

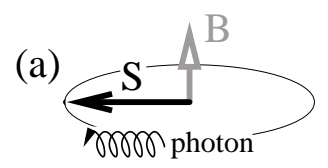

(c)

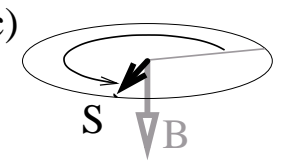

(d)

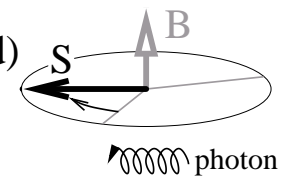

FIG. 5: Sketch of the spin dynamics in the Faraday setup with an oscillating field: (a) The initial measurement of the spin state. (b) In the external field the spin precesses in one direction. (c) When the field changes its sign, the spin precesses in the opposite direction. (d) After a full fieldoscillation time, the spin is again in its initial state.

with the field oscillation frequency $\gamma$. With this Hamiltonian, we can derive the propagator $\Pi(t)$, including a phenomenological relaxation term. Since the experiment should be a continuous-wave experiment, there shall be no correlation between the phase of the magnetic field oscillation, and the absolute time of measurement of the spins. Therefore, we average over the phase $\phi$, and get $\Pi_{\downarrow \downarrow}^{\uparrow \uparrow}(t)=\int_{0}^{2 \pi}(d \phi / 2 \pi) \exp \left[-t / T_{2}+i \omega_{0} / \gamma(\sin (\gamma t+\phi)-\right.$ $\sin \phi)]$. Making use of the Jacobi-Anger expansion,, 19 and the integral representation of the Bessel function, we obtain the power spectrum of the spin-spin correlation function as

$$
S(\omega)=\hbar^{2} \sum_{n=-\infty}^{+\infty}\left[J_{n}\left(\frac{\omega_{0}}{\gamma}\right)\right]^{2} \frac{T_{2}}{1+T_{2}^{2}(\omega+n \gamma)^{2}}
$$

The power spectrum consists of a series of Lorentzian resonances of the width $T_{2}^{-1}$ as plotted in Fig. [6 The different Lorenzian lines in the noise spectrum correspond to multi-photon absorption processes. Several photons of the exciting magnetic field are converted in a single noise quantum with correspondingly increased frequency. The appearance of the multi-resonances is extensively discussed in the context of ESR and NMR, see Ref. 8 and citations therein.

The signal strength of the Faraday spectrum is proportional to the square of Bessel functions $J_{n}$ of the first kind. If the argument of these Bessel functions is of the order of 1 , one can expect approximatively half of the signal magnitude compared to the case of a static magnetic field 16 Therefore, every experimental setup capable to resolve Faraday-rotation fluctuation in a static field should also do so in an oscillating field.

The property of the correlation spectrum, that the resonances appear at multiples of the oscillation frequency $\gamma$ of the external magnetic field rather than the Larmor frequency $\omega_{0}$ offers the possibility to utilize such a measurement for metrology. Since the field oscillation frequency $\gamma$ does not vary locally over the sample size, the width of the resonances is not influenced by locally varying $g$-factors. Therefore a inhomogeneous broadening of 


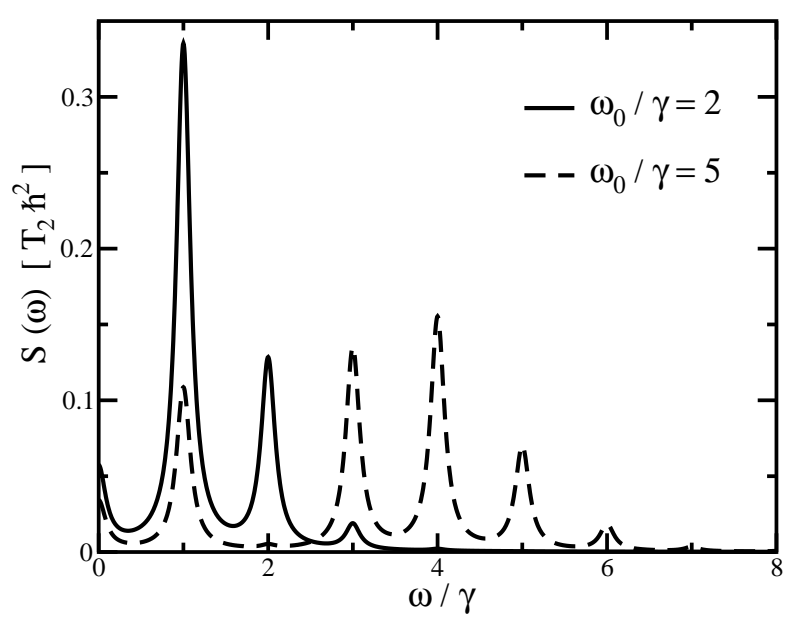

FIG. 6: Spectrum of the spin-spin correlation function for different ratios of magnetic field strength to oscillation frequency. For a higher ratio $\omega_{0} / \gamma$, more resonance lines appear at higher frequencies, while the signal strength decreases. Here, we chose $T_{2}^{-1}=0.1 \gamma$.

the resonance line due to structural or chemical inhomogeneities is avoided. In this sense a Faraday-rotation fluctuation measurement in the presence of an oscillating magnetic field represent a continuous wave realization of an ESR spin-echo experiment.

Such a measurement method could be usefull for samples with weak structural homogeneity, such as chemically synthesized CdSe quantum dots. In these quantum dot arrays, the local variation of the $g$-factors dominate

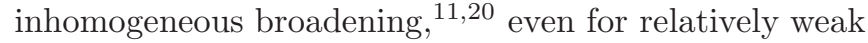
magnetic fields. Unfortunately, the presence of an additional static field component in $z$-direction leads to a shift of the spectrum in Eq. (6). Therefore inhomogeneous line broadening due to hyperfine interaction persist also in the case of an oscillating magnetic field.

While the physical limition of the proposed experiment is that the sample structure must exhibit spin orbit coupling and show the Faraday effect, the generation of the oscillating magnetic field defines the technical limitation. To resolve individual lines, to measure the line width $T_{2}$, the separation of the lines given by the field frequency $\gamma$ must exceed $T_{2}^{-1}$. Further, to get a resonance at $\omega \neq 0$, the field strength $\omega_{0}$ must be comparable to the field frequency $\gamma$. With an experimentally challenging magnetic field of the order of $10 \mathrm{mT}$ at the frequency of the order $100 \mathrm{MHz}$, spin-coherence times down to some tens of ns could be measured. With increasing spin-coherence times the experimental requirements for the oscillating magnetic field relax. Therefore by using a $\mathrm{Rb}$ gas sample, with a spin-coherence time exceeding $100 \mu \mathrm{s}, 14$ such a multi-photon absorption spectrum should be easily to realize. It is worth to mention, that even if the spin decoherence time $T_{2}$ is significantly shorter than the field oscillation frequency, i.e. if the multi-photon resonances are washed out, the dependence of the spectrum on the
Bessel function still persists, 18

In Fig. 7 we plot the spectrum of the spin-spin correlation function $S(\omega)$ as function of $\omega / \gamma$ (noise frequency over field frequency) and $\omega_{0} / \gamma$ (field strength over field frequency). The two horizontal lines indicate the parameters for the spectra shown in Fig. 6. If the time between the two spin measurements gets significantly below the time needed by the spins to close their trajectory, no spin correlation can be measured. Therefore Fig. 7 shows no signal in the parameter range $\omega>\omega_{0}$.

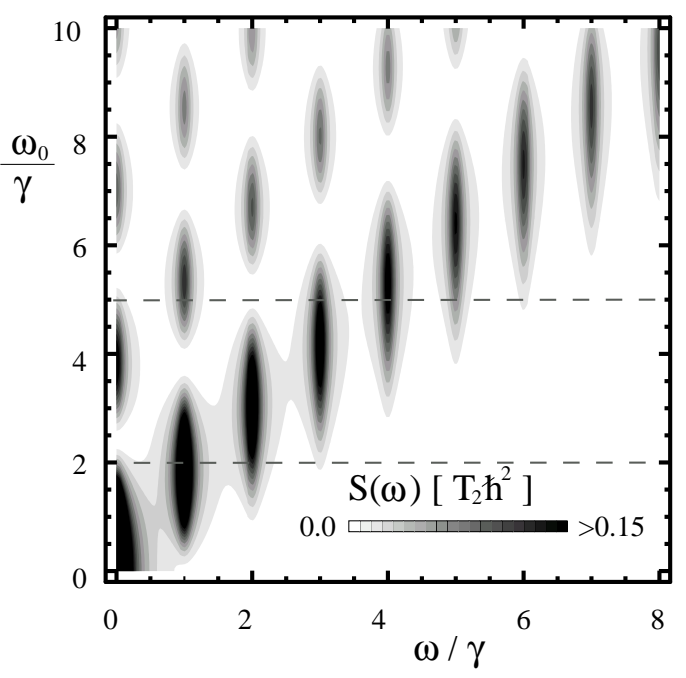

FIG. 7: Spin-spin correlation spectrum, as a function of the ratio $\omega_{0} / \gamma$ and frequency $\omega$ for $T_{2}^{-1}=0.1 \gamma$.

Recently such a "Bessel staircase" as in Fig. [7 was measured by Oliver et $a l_{.21}^{21}$ and M. Sillanpää et $a l ., 22$ not in a spin system but for the excitation probability in a strongly-driven superconducting flux/charge qubit used as as Mach-Zehnder interferometer. The main physical difference between Faraday-rotation fluctuation spectroscopy and the Qubit case is, that in latter an average quantity is measured, i.e. the probability for excitation, while in former the correlation function is important. However, since in lowest order perturbation theory, the transition probability of the Qubit states can also be represented as a correlation function, 18 the close correspondence of these two different experiments becomes immanent.

\section{CONCLUSIONS}

In conclusion, we present an elementary theoretical explanation of recent experiments measuring spin noise in the presence of a static magnetic field via the Faraday-induced fluctuations. Furthermore, we predict the Faraday-rotation fluctuation spectrum if one applies an oscillating magnetic field instead of a static one. Such an experiment should offer an experimental easy possibility to observe multi-photon absorption processes. Fur- 
thermore by such a measurement one should be capable to measure spin-relaxation rates down to some 10th of ns, without inhomogeneous broadening due to structural or chemical inhomogeneities, whereas the influence of hy- perfine interaction will persist.

We thank D. Hägele, B. Kubala, M. Oestreich, and D. Urban for discussions. This work was supported by the DFG under SFB 491 and GRK 726.
1 D. Loss and D. P. DiVincenzo, Phys. Rev. A 57, 120 (1998).

2 I. Zutic, J. Fabian, and S. Das Sarma, Rev. Mod. Phys. 76, 323 (2004).

3 I. A. Merkulov, A. L. Efros, and M. Rosen, Phys. Rev. B, 65, 205309; A. V. Khaetskii, D. Loss, and L. Glazman, Phys. Rev. Lett. 88, 186802 (2002); A. V. Khaetskii and Y. V. Nazarov, Phys. Rev. B 64, 125316 (2001).

4 T. Fujisawa, D. G. Austing, Y. Tokura, Y. Hirayama, and S. Tarucha, Phys. Rev. Lett. 88, 236802 (2002).

5 J. M. Elzerman, R. Hanson, L. H. Willems van Beveren, B. Witkamp, L. M. K. Vandersypen, and L. P. Kouwenhoven, Nature 430, 431 (2004).

6 E. L. Hahn, Phys. Rev. 80, 580 (1950).

7 R. J. Blume, Phys. Rev. 109, 1867 (1958).

8 M. Kälin, I. Gromov, and A. Schweiger, J. of Magnetic Resonance 160, 166 (2003).

9 R. J. Epstein, D. T. Fuchs, W. V. Schoenfeld, P. M. Petroff, and D. D. Awschalom, Appl. Phys. Lett. 78, 733 (2001).

10 R. I. Dzhioev, V. L. Korenev, I. A. Merkulov, B. P. Zakharchenya, D. Gammon, Al. L. Efros, and D. S. Katzer, Phys. Rev. Lett. 88, 256801 (2002).

11 N. P. Stern, M. Poggio, M. H. Bartl, E. L. Hu, G. D. Stucky, and D. D. Awschalom, cond-mat/0507699
12 J. A. Gupta, D. D. Awschalom, X. Peng, and A. P. Alivisatos, Phys. Rev. B 59, R10421 (1999).

13 E. B. Aleksandrov and V. S. Zapassky, Zh. Eksp. Teor. Fiz. 81, 132 (1981).

14 S. A. Crooker, D. G. Rickel, A. V. Balatsky, and D. L. Smith, Nature 431, 49 (2004).

15 T. Mitsui, Phys. Rev. Lett. 84, 5292 (2000).

16 M. Oestreich, M. Römer, R. J. Haug, and D. Hägele, Phys. Rev. Lett. 95, 216603 (2005).

17 The factor of $1 / 2$ in Eq. (2) corrects for a double counting during this procedure.

18 D. M. Berns, W. D. Oliver, S. O. Valenzuela, A. V. Shytov, K. K. Berggren, L. S. Levitov, and T. P. Orlando, cond-mat/0606271

19 Handbook of Mathematical Functions, edited by M. Abramowitz and I. A. Stegun (National Bureau of Standards, 1972), p. 361.

20 J. A. Gupta, R. Knobel, N. Samarth, and D. D. Awschalom, Science 292, 2458 (2001).

21 W. D. Oliver, Y. Yu, J. C. Lee, K. K. Berggren, L. S. Levitov, and T. P. Orlando, Science 310 1653, (2005).

22 M. Sillanpää, T. Lehtinen, A. Paila, Y. Makhlin, and P. Hakonen, cond-mat/0510559. 